ఠ

\title{
Mutational analysis on gastric, duodenal, bone, and mediastinal lymph node metastases and blood from a case of primary lung adenocarcinoma
}

This article was published in the following Dove Press journal: OncoTargets and Therapy

\author{
Xin Yang \\ Rui Chen \\ Chen Wu \\ Weiqing Zhao \\ Mei Ji \\ Department of Oncology, The Third \\ Affiliated Hospital of Soochow \\ University, Changzhou 213003, China
}

Correspondence: Mei Ji

Department of Oncology, The Third Affiliated Hospital of Soochow University, No 185 Juqian Road, Tianning District, Changzhou 213003, China Email zlkjimei@I63.com

\begin{abstract}
Rapid metastasis contributes significantly to the high mortality rates in lung adenocarcinoma. The present study reports a rare case of primary lung adenocarcinoma with concomitant gastric, duodenal, bone, and mediastinal lymph node metastases. A large gene panel based on the next-generation sequencing was used to detect gene mutations in different metastatic sites and blood. The results showed that the gene mutation spectrums among different metastatic foci were roughly similar. The mutation abundance was highest in mediastinal lymph nodes. Unique mutation sites were detected only in mediastinal lymph nodes, bone, and gastric metastatic foci. Despite partial heterogeneity, there are currently no applicable targeted drugs to adopt. In addition, tumor mutation burden (TMB) showed that blood, gastric, bone, and mediastinal lymph node metastases were all TMB-High, while the duodenal metastasis was TMB-Low. This study is the first to report a rare case of newly diagnosed lung adenocarcinoma with concomitant gastrointestinal metastases and to perform mutation analyses on all metastatic foci. Large sample sizes with similar cases are required to gain deeper insights.
\end{abstract}

Keywords: lung adenocarcinoma, gastric metastasis, duodenal metastasis, TMB

\section{Introduction}

In 2016, Cancer Statistics reported that lung cancer continues to be a malignant tumor with high morbidity and mortality. ${ }^{1}$ Most patients have already developed distant metastases when they first seek treatment. The common metastatic locations are the brain, liver, adrenal glands, and bones. Gastrointestinal metastasis of lung adenocarcinoma is rare. Recent large-sample retrospective clinical studies have shown that its clinical incidence is approximately $0.26 \%-0.33 \% .^{2,3}$ Lung adenocarcinoma with concomitant gastric and duodenal metastases at initial diagnosis is extremely rare, and so far we have found no relative case reports in the literature. Many recent studies have discovered inconsistent gene mutations between the primary tumor and metastases within the same individual. ${ }^{4-6}$ However, mutational analysis among different metastatic foci in the same individual is rare. This paper reports the case of newly diagnosed primary lung adenocarcinoma with concomitant gastric, duodenal, bone, and mediastinal lymph node metastases. A gene panel based on the next-generation sequencing (NGS) was used to detect gene mutations among different metastatic foci and blood.

\section{Case presentation}

A 59-year-old male patient was referred to our hospital with a 1 week history of right upper limb pain and stomach discomfort. The patient had a 60-pack-year smoking 

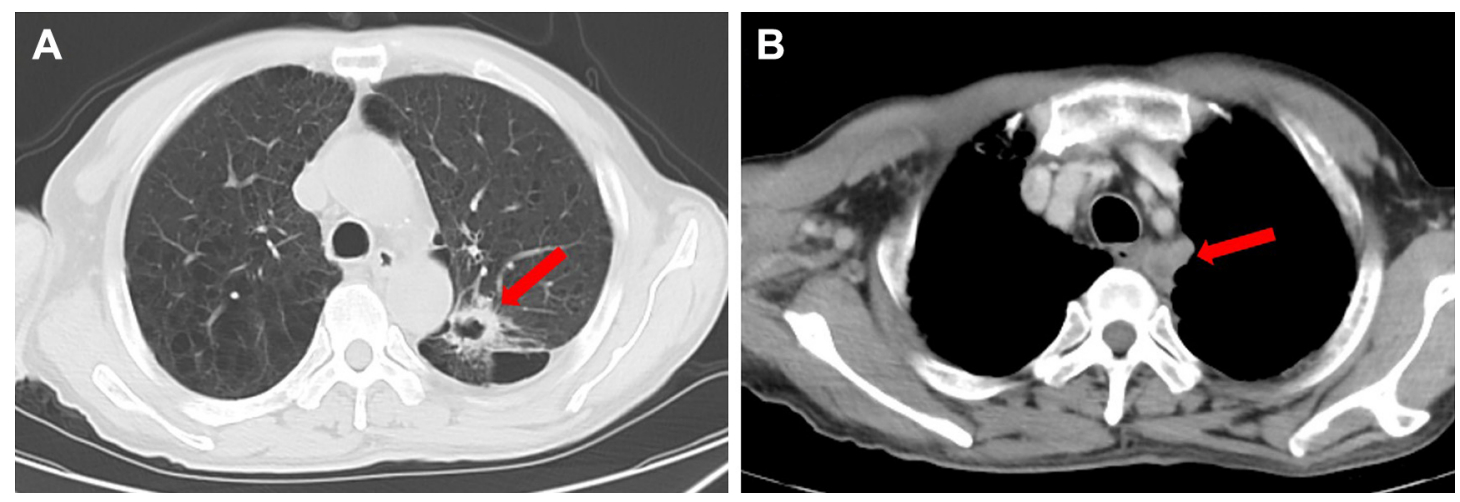

Figure I (A) Chest computed tomography revealed a lobulated soft tissue $(2.7 \times 2.3 \mathrm{~cm}$, arrow) with an irregular hollow shadow in the posterior segment of the upper left lung lobe. (B) Mediastinal and perihilar lymph node metastases were noted (arrow).

history with no family history of cancer. He worked in an industrial wastewater treatment plant for many years. Related examinations were performed upon admission, which showed abnormally increased tumor indicators (CEA: 75.95 ng/mL; CYFRA21-1: 11.62 ng/mL; CA72-4: 17.89 U/mL; CA-125: $45.39 \mathrm{U} / \mathrm{mL}$; and CA-153: $62.97 \mathrm{U} / \mathrm{mL})$. Chest computed tomography revealed a lobulated soft tissue mass measuring $2.7 \times 2.3 \mathrm{~cm}$ in the posterior segment of the upper left lung lobe, and an irregular hollow shadow existed in the foci (Figure 1A). Mediastinal and perihilar lymph node metastases were noted (Figure 1B). Furthermore, the right upper humeral bone metastasis combined with a pathological bone fracture was observed (Figure 2A), which was confirmed by ${ }^{99 \mathrm{~m}} \mathrm{Tc}$ bone scan. Brain magnetic resonance imaging result was negative. Because of the restricted location of pulmonary lesions and the presence of pulmonary bullae in the surrounding tissues, computed tomography-guided transthoracic needle lung biopsy or electron tracheoscopeguided transbronchial lung biopsy was considered too risky to perform. Therefore, the metastasis from the upper right
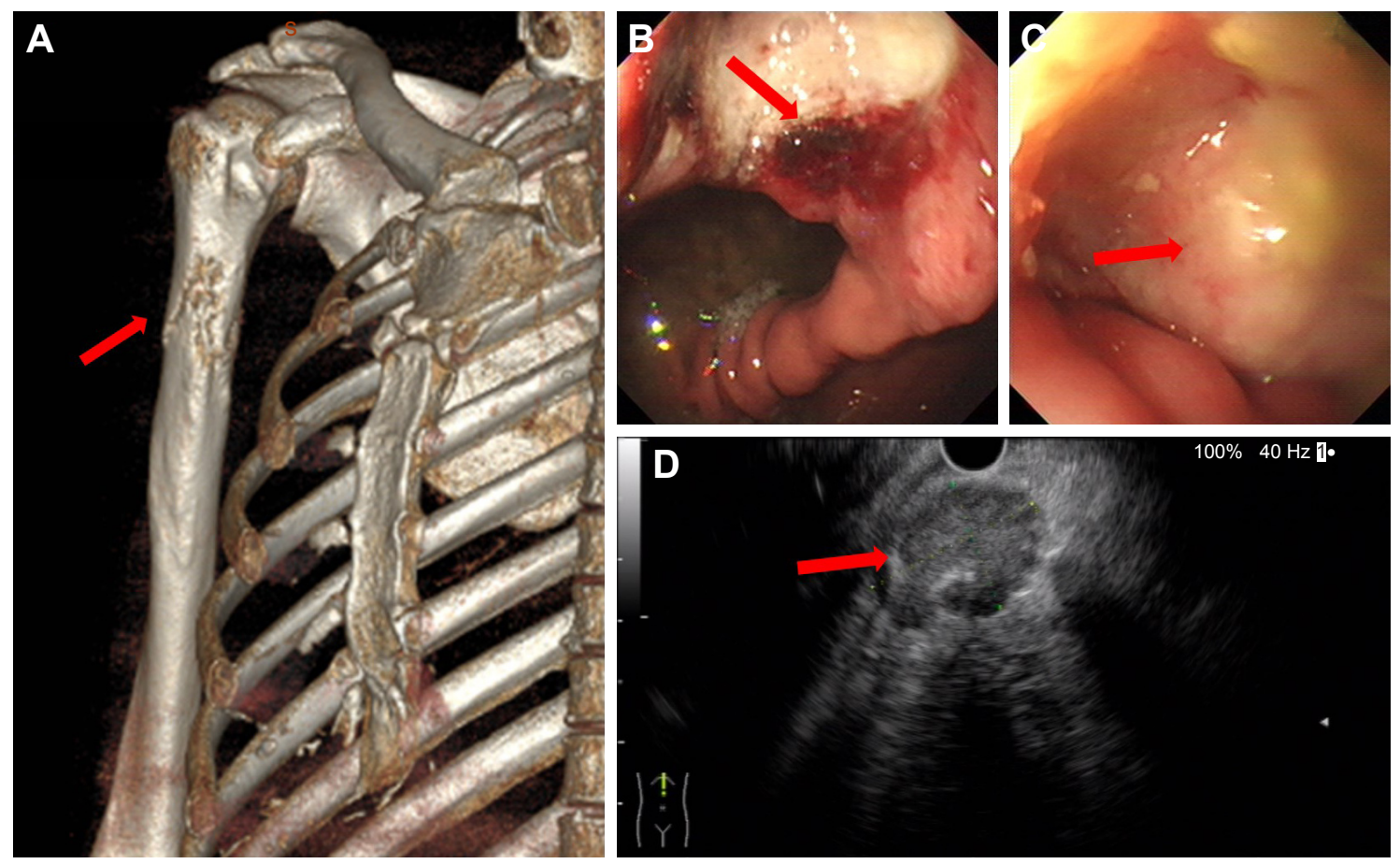

Figure 2 (A) Three-dimensional reconstruction of computed tomography image revealed that the right upper humeral bone metastasis was combined with a pathological bone fracture (arrow). (B) Gastroscopy revealed an ulcer (arrow) of approximately $2 \times 2 \mathrm{~cm}$ located in posterior wall of gastric corpus. (C) A rough uplift (arrow) of $1.5 \times 2.0 \mathrm{~cm}$ was observed in the junction of duodenal bulb and descending part. (D) Endoscopic ultrasound-guided fine needle aspirate was performed on mediastinal lymph nodes (arrow). 
humeral segment was biopsied. The pathological results showed poorly differentiated metastatic carcinoma. Immunohistochemistry showed that the tumor was AE1/AE3 (+), TTF-1 (+), CK7 (+), Napsin A ( \pm ), CK5/6 ( \pm$),$ P40 (-), CAIX $(+)$, and CD10 (-), which met the criteria for poorly differentiated metastatic carcinoma which tended to be pulmonary in origin. Gastroscopy revealed an ulcer of approximately $2 \times 2 \mathrm{~cm}$ located in posterior wall of gastric corpus, and it was covered with white mucus and surrounded by congestion and edema (Figure 2B). In addition, a rough uplift of $1.5 \times 2.0 \mathrm{~cm}$ was observed at the junction of the duodenal bulb and the descending part, leaving the bulbar lumen deformed and narrow (Figure 2C). Next, an endoscopic ultrasound-guided fine needle aspirate was performed on the mediastinal lymph nodes (Figure 2D). These pathological diagnoses of biopsy tissues were all metastatic poorly differentiated adenocarcinomas. Immunohistochemistry demonstrated TTF-1 (+), CK7 (+), Napsin A (+), CK20 (-), CA19-9 (-), and Ki67 (approximately $50 \%+$ ), which supported the assumption of lung adenocarcinoma that had metastasized to stomach, duodenum, and mediastinal lymph nodes.

A large gene panel based on the NGS was used to detect gene mutations among the four tissue and blood samples. The NGS genetic testing panel (OncoScreen Plus ${ }^{\mathrm{TM}}$, Burning Rock Company, Guangzhou, China) ${ }^{7}$ involves a modified Roche/Nimblegen SeqCap EZ (Roche, Madison, WI, USA) covering 520 genes that are closely associated with cancer pathogenesis and targeted therapy, including whole exon regions of 312 genes and hotspot mutation regions (exons, introns, and promoter regions) of 208 genes. The large panel can comprehensively and accurately detect variations involving gene mutations, amplifications, and fusions with clear clinical relevance to tumors. Unfortunately, the results showed no detected mutations in core genes associated with solid tumors (AKT1, ALK, BRAF, BRCA1, BRCA2, EGFR, ERBB2, ESR1, HRAS, KIT, KRAS, MET, NRAS, PDGFRA, $P T E N, R E T$, and ROS1). All the detected mutation genes and their abundance, the tumor mutation burden (TMB), and microsatellite stability types are shown in Figure 3A. Among all metastatic foci, the mutation abundance in the mediastinal lymph nodes $(p<0.001)$, stomach $(p=0.001)$, and bone ( $p=0.018$ ) were significantly higher than that in blood, as shown in Figure 3B. (Results are expressed as mean \pm SD. Comparisons of the quantitative data were performed using one-way analysis of variance. The $p$-value of $<0.05$ based on the 2-sided test is considered statistically significant.) Unique mutation sites were only detected in mediastinal lymph nodes (EPHA3 p.E647K, ABL2 p.L1048V, RANBP2 p.D1926H,
FBXW7 p.Ser678, and NEGR1 p.N107D), stomach (NTRK1 p.S439F, KMT2C p.D1234Y, JUN p.E238K, JUN p.G139R, MLH1 p.E430D, RAD50 p.Q199K, RPTOR p.R348G, RBM10 p.G567S, PBRM1 c.237-1G>A, PRKDC p.D3830Y, and HNF1A p.Trp 165), and bone (TAF1 p.Lys667fs) metastases. Genetic testing showed that the gene mutation spectrums of the 4 tissue samples were roughly similar, which might indirectly confirm that all tissue samples were lung adenocarcinoma metastatic foci.

Additionally, TMB values for blood, mediastinal lymph nodes, bone, and gastric metastatic foci were significantly higher than that of the duodenal metastatic focus. Therefore, blood, mediastinal lymph nodes, bone, and gastric metastatic foci were evaluated as TMB-High, and the duodenal metastatic focus was evaluated as TMB-Low (TMB $\geq 20$ was defined as TMB-High, $10<$ TMB $<20$ as TMB-Medium, and $\mathrm{TMB} \leq 10$ as TMB-Low).

According to the National Comprehensive Cancer Network clinical practice guidelines for non-small-cell lung cancer (2017, Version 4), the patient would receive 6 cycles of pemetrexed $\left(500 \mathrm{mg} / \mathrm{m}^{2}\right.$ ) and carboplatin (AUC $6.0 \mathrm{mg} / \mathrm{mL}$ min) administered at 21 day intervals as first-line therapy. Although TMB was high in 4 metastatic foci, PD-L1 expression was negative in all tissues. Thus, anti-PD-1 immunotherapy would be applied after first-line therapy.

\section{Discussion}

Tumor metastasis is the process by which cancer cells disperse from a primary site and progressively colonize distant organs. Certain heterogeneity exists between primary tumor and metastases, and gene mutation probabilities in metastases are significantly higher than that in the primary tumor. ${ }^{8}$ The metastatic process of tumor cells is affected by both the internal immune microenvironment in different metastatic sites and the external intervention of different chemotherapies, molecular targeted therapies, or immune therapy regimens, which altogether result in the heterogeneity of metachronous metastatic foci. ${ }^{9}$ Currently, few studies have focused on the heterogeneity among different metastatic foci in the same individual, especially those in rare metastatic sites. This study, for the first time, reported a rare case of lung adenocarcinoma with concomitant gastric and duodenal metastases. In addition, NGS was performed to detect 520 tumor-associated gene mutations in all metastatic foci and blood. Unfortunately, the mutation analyses in this case suggested that the patient could not benefit from any currently available targeted drugs. However, we found that the mutation spectrums between the metastatic foci and blood were 
roughly similar. The main reasons for this were as follows: 1) this patient worked in an industrial wastewater treatment plant for many years. Industrial pollution was considered as an important cause of disease, and the patient was also a heavy smoker. Based on relevant studies, these factors might have caused the low percentage of EGFR mutations and high TMB levels. ${ }^{10,11}$ 2) The patient already had multiple metastases upon initial diagnosis and had previously received no medical interventions. So, tumor cells mainly followed a natural metastatic pattern.

\begin{tabular}{|c|c|c|c|c|c|}
\hline Mutation & Blood & Bone & Duodenum & Lymph nodes & Stomach \\
\hline TP53 p.Ser116fs & $6.28 \%$ & $13.30 \%$ & $\mathrm{~N} / \mathrm{A}$ & $57.59 \%$ & $2.92 \%$ \\
\hline STK11 p.GIn220* & $5.67 \%$ & $18.02 \%$ & $\mathrm{~N} / \mathrm{A}$ & $78.52 \%$ & $20.71 \%$ \\
\hline PIK3CA p.E542K & $3.51 \%$ & $7.32 \%$ & $2.36 \%$ & $28.99 \%$ & $13.77 \%$ \\
\hline PAK3 p.M282I & $5.58 \%$ & $28.62 \%$ & N/A & $71.10 \%$ & $28.09 \%$ \\
\hline AKT2 p.F470L & $3.88 \%$ & $12.44 \%$ & $\mathrm{~N} / \mathrm{A}$ & $25.61 \%$ & $12.55 \%$ \\
\hline SMAD4 p.R496H & $6.04 \%$ & $17.68 \%$ & $\mathrm{~N} / \mathrm{A}$ & $57.05 \%$ & $20.15 \%$ \\
\hline FLT3 c.2654-2A>T & $3.98 \%$ & $12.92 \%$ & $\mathrm{~N} / \mathrm{A}$ & $53.55 \%$ & $18.16 \%$ \\
\hline CDKN2A p.G101W & $3.11 \%$ & $18.72 \%$ & $\mathrm{~N} / \mathrm{A}$ & $67.44 \%$ & $12.29 \%$ \\
\hline NTRK1 p.S439F & N/A & N/A & $\mathrm{N} / \mathrm{A}$ & N/A & $8.66 \%$ \\
\hline FGF3 p.R164C & $1.91 \%$ & $6.40 \%$ & $\mathrm{~N} / \mathrm{A}$ & $38.61 \%$ & $5.83 \%$ \\
\hline HGF p.D290G & $4.35 \%$ & $8.92 \%$ & $\mathrm{~N} / \mathrm{A}$ & $28.44 \%$ & $31.85 \%$ \\
\hline HGF p.C70R & $4.72 \%$ & $9.36 \%$ & $\mathrm{~N} / \mathrm{A}$ & $23.04 \%$ & $28.37 \%$ \\
\hline KEAP1 p.R320L & $4.67 \%$ & $11.93 \%$ & $\mathrm{~N} / \mathrm{A}$ & $71.79 \%$ & $21.65 \%$ \\
\hline EPHA3 p.E647K & $\mathrm{N} / \mathrm{A}$ & $\mathrm{N} / \mathrm{A}$ & $\mathrm{N} / \mathrm{A}$ & $18.02 \%$ & $\mathrm{~N} / \mathrm{A}$ \\
\hline EPHA5 p.A241G & $1.82 \%$ & $6.88 \%$ & $\mathrm{~N} / \mathrm{A}$ & $31.04 \%$ & $\mathrm{~N} / \mathrm{A}$ \\
\hline EPHA7 p.Y132H & $2.33 \%$ & $8.94 \%$ & $\mathrm{~N} / \mathrm{A}$ & $57.78 \%$ & $18.28 \%$ \\
\hline KMT2C p.Cys473* & $2.55 \%$ & $6.55 \%$ & $\mathrm{~N} / \mathrm{A}$ & $45.43 \%$ & $8.18 \%$ \\
\hline KMT2C p.D1234Y & $\mathrm{N} / \mathrm{A}$ & $\mathrm{N} / \mathrm{A}$ & $\mathrm{N} / \mathrm{A}$ & $\mathrm{N} / \mathrm{A}$ & $4.38 \%$ \\
\hline KMT2D p.R3714W & $4.23 \%$ & $14.78 \%$ & $\mathrm{~N} / \mathrm{A}$ & $30.26 \%$ & $17.71 \%$ \\
\hline KMT2D p.D992H & $5.36 \%$ & $12.06 \%$ & $\mathrm{~N} / \mathrm{A}$ & $30.58 \%$ & $16.37 \%$ \\
\hline ABL2 p.P792L & $5.06 \%$ & $7.84 \%$ & $2.03 \%$ & $19.94 \%$ & $17.00 \%$ \\
\hline ABL2 p.L1048V & $\mathrm{N} / \mathrm{A}$ & $\mathrm{N} / \mathrm{A}$ & $\mathrm{N} / \mathrm{A}$ & $21.08 \%$ & $\mathrm{~N} / \mathrm{A}$ \\
\hline INHBA p.R379L & $4.61 \%$ & $11.82 \%$ & $\mathrm{~N} / \mathrm{A}$ & $61.27 \%$ & $16.13 \%$ \\
\hline GNAS p.S380F & $2.88 \%$ & $4.91 \%$ & $\mathrm{~N} / \mathrm{A}$ & $24.02 \%$ & $13.74 \%$ \\
\hline PREX2 p.C94F & $6.32 \%$ & $12.07 \%$ & $2.15 \%$ & $40.85 \%$ & $13.05 \%$ \\
\hline JUN p.E238K & $\mathrm{N} / \mathrm{A}$ & $\mathrm{N} / \mathrm{A}$ & $\mathrm{N} / \mathrm{A}$ & $\mathrm{N} / \mathrm{A}$ & $10.03 \%$ \\
\hline JUN p.G139R & $\mathrm{N} / \mathrm{A}$ & $\mathrm{N} / \mathrm{A}$ & $\mathrm{N} / \mathrm{A}$ & $\mathrm{N} / \mathrm{A}$ & $8.39 \%$ \\
\hline LRP1B p.D344N & $3.50 \%$ & $8.20 \%$ & $\mathrm{~N} / \mathrm{A}$ & $\mathrm{N} / \mathrm{A}$ & $9.73 \%$ \\
\hline MLH1 p.E430D & $\mathrm{N} / \mathrm{A}$ & $\mathrm{N} / \mathrm{A}$ & $\mathrm{N} / \mathrm{A}$ & $\mathrm{N} / \mathrm{A}$ & $9.53 \%$ \\
\hline TCF7L2 p.D421G & $4.11 \%$ & $4.73 \%$ & $\mathrm{~N} / \mathrm{A}$ & $28.09 \%$ & $9.18 \%$ \\
\hline TAF1 p.Lys667fs & $\mathrm{N} / \mathrm{A}$ & $4.42 \%$ & $\mathrm{~N} / \mathrm{A}$ & $\mathrm{N} / \mathrm{A}$ & $\mathrm{N} / \mathrm{A}$ \\
\hline RAD50 p.Q199K & $\mathrm{N} / \mathrm{A}$ & $\mathrm{N} / \mathrm{A}$ & $\mathrm{N} / \mathrm{A}$ & $\mathrm{N} / \mathrm{A}$ & $8.43 \%$ \\
\hline RANBP2 p.D1926H & $\mathrm{N} / \mathrm{A}$ & N/A & $\mathrm{N} / \mathrm{A}$ & $31.82 \%$ & $\mathrm{~N} / \mathrm{A}$ \\
\hline RPTOR p.R348G & $\mathrm{N} / \mathrm{A}$ & $\mathrm{N} / \mathrm{A}$ & $\mathrm{N} / \mathrm{A}$ & $\mathrm{N} / \mathrm{A}$ & $7.62 \%$ \\
\hline RBM10 p.G567S & $\mathrm{N} / \mathrm{A}$ & $\mathrm{N} / \mathrm{A}$ & $\mathrm{N} / \mathrm{A}$ & $\mathrm{N} / \mathrm{A}$ & $6.62 \%$ \\
\hline CDC73 p.W492C & $2.37 \%$ & $7.79 \%$ & $\mathrm{~N} / \mathrm{A}$ & $49.25 \%$ & $6.60 \%$ \\
\hline PBRM1 c.237-1G>A & $\mathrm{N} / \mathrm{A}$ & $\mathrm{N} / \mathrm{A}$ & $\mathrm{N} / \mathrm{A}$ & $\mathrm{N} / \mathrm{A}$ & $6.44 \%$ \\
\hline PRKDC p.D3830Y & N/A & N/A & $\mathrm{N} / \mathrm{A}$ & $\mathrm{N} / \mathrm{A}$ & $5.70 \%$ \\
\hline HNF1A p.Trp165* & $\mathrm{N} / \mathrm{A}$ & N/A & $\mathrm{N} / \mathrm{A}$ & $\mathrm{N} / \mathrm{A}$ & $4.22 \%$ \\
\hline FBXW7 p.Ser678* & $\mathrm{N} / \mathrm{A}$ & $\mathrm{N} / \mathrm{A}$ & $\mathrm{N} / \mathrm{A}$ & $31.28 \%$ & $\mathrm{~N} / \mathrm{A}$ \\
\hline NEGR1 p.N107D & N/A & N/A & $N / A$ & $24.83 \%$ & N/A \\
\hline TMB (/Mb) & High (29.4) & High (31) & Low (4.8) & High (33.3) & High (37.3) \\
\hline MS status & & MSS & MSS & MSS & MSS \\
\hline
\end{tabular}

Figure 3 (Continued) 


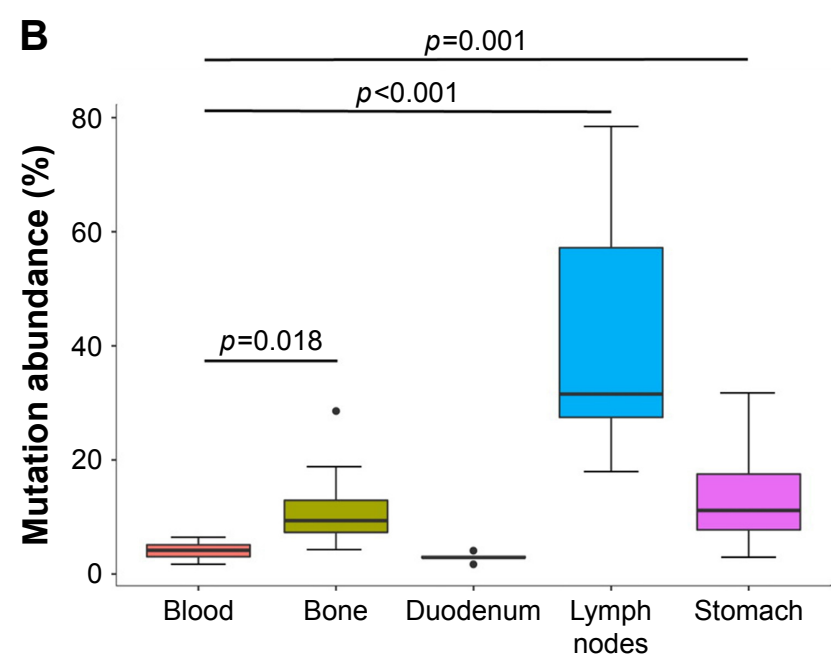

Figure 3 (A) Mutational analysis on gastric, duodenal, bone, mediastinal lymph node metastases, and blood. Different colors represent unique mutation sites in each location. (B) The mutation abundance on all metastatic foci and blood. The $p$-value of $<0.05$ based on the 2 -sided test is considered to be statistically significant.

Note: $*$ nonsense mutation.

Furthermore, we found that the mutation abundance was highest in mediastinal lymph nodes was highest among all metastases. In such cases, where the primary foci cannot be biopsied, mediastinal lymph node biopsy might be preferentially considered. Although the mutation abundance of liquid biopsy was significantly lower than that of tumor tissue biopsies, its TMB level was equivalent to that of tumor biopsies. The liquid biopsy technology based on NGS might be an appropriate alternative for TMB analysis in this case. However, the greatest disadvantage of liquid biopsy technology is its relatively low sensitivity. ${ }^{12}$

Clinical studies have indicated that higher TMB may yield better response rate to PD-1 inhibition treatment. ${ }^{13}$ The TMB results obtained from the NGS large panel or WES were highly correlated, confirming that selective sequencing of limited regions could sufficiently provide a full understanding of mutational burdens for predicting the potential anti-PD-1/ PD-L1 therapeutic efficacy. ${ }^{14}$ Evaluating the blood, mediastinal lymph nodes, bone, and gastric metastases in this patient indicated that the patient was TMB-High, yet the duodenal metastatic focus presented as TMB-Low. The inconsistency is difficult to explain but may be associated with the absolute level and different metastatic routes of tumor cells.

In summary, gastrointestinal metastasis in newly diagnosed lung adenocarcinoma is rare in clinical practice. Mutation analyses on all metastatic foci and blood performed in the present case helped us realize that the gene mutation analyses can yield comprehensive information for targeted therapy guidance and can have certain reference value for clinically diagnosing metastatic foci. More in-depth studies need to be performed in large sample sizes of patients with similar rare disease.

\section{Informed consent}

Written informed consent was obtained from the patient for publication of this manuscript and any accompanying images.

\section{Acknowledgment}

This work was supported by grants from the Natural Science Youth Foundation of China (81501971 to Xin Yang, 31700792 to $\mathrm{Chen} \mathrm{Wu}$ ), the Natural Science Youth Foundation of Jiangsu Province (BK20150252 to Xin Yang), the Human Resource Summit Grant of Jiangsu Province (WSW-142 to Xin Yang), the Youth Medical Professionals Foundation of Jiangsu Province (QNRC2016279 to Xin Yang), and Changzhou Science and Technology Project (Applied Based Research, No CJ20159021 to Chen Wu).

\section{Disclosure}

The authors report no conflicts of interest in this work.

\section{References}

1. Siegel RL, Miller KD, Jemal A. Cancer statistics, 2016. CA Cancer J Clin. 2016;66(1):7-30.

2. Lee PC, Lo C, Lin MT, Liang JT, Lin BR. Role of surgical intervention in managing gastrointestinal metastases from lung cancer. World $J$ Gastroenterol. 2011;17(38):4314-4320.

3. Taira N, Kawabata T, Gabe A, et al. Analysis of gastrointestinal metastasis of primary lung cancer: clinical characteristics and prognosis. Oncol Lett. 2017;14(2):2399-2404.

4. Italiano A, Vandenbos FB, Otto J, et al. Comparison of the epidermal growth factor receptor gene and protein in primary non-small-cell-lung cancer and metastatic sites: implications for treatment with EGFRinhibitors. Ann Oncol. 2006;17(6):981-985.

5. Daniele L, Cassoni P, Bacillo E, et al. Epidermal growth factor receptor gene in primary tumor and metastatic sites from non-small cell lung cancer. J Thorac Oncol. 2009;4(6):684-688. 
6. Han C, Ma J, Zhao J, Zhou Y, Jing W, Zou H. EGFR mutations, gene amplification, and protein expression and KRAS mutations in primary and metastatic tumors of nonsmall cell lung cancers and their clinical implications: a meta-analysis. Cancer Invest. 2011;29(9):626-634.

7. Stoy SP, Segal JP, Mueller J, et al. Feasibility of endobronchial ultrasound-guided transbronchial needle aspiration cytology specimens for next generation sequencing in non-small-cell lung cancer. Clin Lung Cancer. 2018;19(3):230-238.e2.

8. Robinson DR, Wu YM, Lonigro RJ, et al. Integrative clinical genomics of metastatic cancer. Nature. 2017;548(7667):297-303.

9. Gerlinger M, Rowan AJ, Horswell S, et al. Intratumor heterogeneity and branched evolution revealed by multiregion sequencing. $N$ Engl J Med. 2012;366(10):883-892.

10. Tseng CH, Chiang CJ, Tseng JS, et al. EGFR mutation, smoking, and gender in advanced lung adenocarcinoma. Oncotarget. 2017;8(58): 98384-98393.
11. Alexandrov LB, Ju YS, Haase K, et al. Mutational signatures associated with tobacco smoking in human cancer. Science. 2016;354(6312): 618-622.

12. Wan JCM, Massie C, Garcia-Corbacho J, et al. Liquid biopsies come of age: towards implementation of circulating tumour DNA. Nat Rev Cancer. 2017;17(4):223-238.

13. Yarchoan M, Hopkins A, Jaffee EM. Tumor mutational burden and response rate to PD-1 inhibition. $N$ Engl J Med. 2017;377(25): 2500-2501.

14. Rizvi H, Sanchez-Vega F, La K, et al. Molecular determinants of response to anti-programmed cell death (PD)-1 and anti-programmed death-ligand (PD-L)-ligand 1 blockade in patients with non-small-cell lung cancer profiled with targeted next-generation sequencing. J Clin Oncol. 2018;36(7):633-641.
OncoTargets and Therapy

\section{Publish your work in this journal}

OncoTargets and Therapy is an international, peer-reviewed, open access journal focusing on the pathological basis of all cancers, potential targets for therapy and treatment protocols employed to improve the management of cancer patients. The journal also focuses on the impact of management programs and new therapeutic agents and protocols on

\section{Dovepress}

patient perspectives such as quality of life, adherence and satisfaction. The manuscript management system is completely online and includes a very quick and fair peer-review system, which is all easy to use. Visit http://www.dovepress.com/testimonials.php to read real quotes from published authors. 